\title{
Synergistic cycles of protease activity and inflammation via PPARY degradation in chronic obstructive pulmonary disease
}

\author{
Nakwon Kwak ${ }^{1,2}$, Kyoung-Hee Lee ${ }^{1}$, Jisu Woo', Jiyeon Kim', Chang-Hoon Lee ${ }^{1,2}$ and Chul-Gyu Yoo ${ }^{1,2}$
}

\begin{abstract}
Inflammation, oxidative stress, and protease-antiprotease imbalance have been suggested to be a pathogenic triad in chronic obstructive pulmonary disease (COPD). However, it is not clear how proteases interact with components of inflammatory pathways. Therefore, this study aimed to evaluate the effect of neutrophil elastase (NE) on lipopolysaccharide (LPS)-induced interleukin 8 (IL-8) production and determine the molecular mechanism in human bronchial epithelial cells (HBECs). Immortalized bronchial epithelial cells and primary HBECs were used to investigate the impact of NE on LPS-induced IL-8 production. The molecular mechanism by which NE modulated LPS-induced IL-8 production was confirmed in elastase-treated C57BL/6 mice and primary HBECs obtained from COPD patients and healthy controls. The results showed that NE treatment synergistically augmented LPS-induced IL-8 production in both immortalized bronchial epithelial cells and primary HBECs. NE partially degraded peroxisome proliferator-activated receptor gamma (PPARY), which is known to regulate IL-8 production in the nucleus. Treatment with a PPARY agonist and overexpression of PPARY reversed the NE-induced synergistic increase in LPS-induced IL-8 production. Moreover, PPARY levels were lower in lung homogenates and lung epithelial cells from elastase-treated mice than in those from saline-treated mice. In accordance with the findings in mice, PPARy levels were lower in primary HBECs from COPD patients than in those from healthy never-smokers or healthy smokers. In conclusion, a vicious cycle of mutual augmentation of protease activity and inflammation resulting from PPARY degradation plays a role in the pathogenesis of COPD.
\end{abstract}

\section{Introduction}

Chronic obstructive pulmonary disease (COPD) is a progressive inflammatory disease characterized by persistent respiratory symptoms and airflow limitations ${ }^{1}$. The pathogenesis of COPD is complex, and inflammation, oxidative stress, and protease-antiprotease imbalance have been suggested to be a pathogenic triad in $\mathrm{COPD}^{2}$. Within this triad, the most well-known type of pathogenesis associated with COPD is chronic inflammation

Correspondence: Chul-Gyu Yoo (cgyoo@snu.ac.kr)

${ }^{1}$ Division of Pulmonary and Critical Care Medicine, Department of Internal Medicine, Seoul National University Hospital, Seoul, South Korea

${ }^{2}$ Department of Internal Medicine, Seoul National University College of Medicine, Seoul, South Korea

These authors contributed equally: Nakwon Kwak, Kyoung-Hee Lee caused by exposure to noxious irritants, including cigarette smoke (CS). The inhalation of CS activates patternrecognition receptors, including toll-like receptors (TLRs), stimulating an innate immune response that leads to the recruitment of neutrophils and macrophages to the lungs ${ }^{3}$ and activates airway epithelial cells ${ }^{3}$. Activation of these cells triggers the release of various inflammatory cytokines, such as interleukin 8 (IL-8), which acts as a chemoattractant for neutrophils; these recruited neutrophils perpetuate chronic inflammation in $\mathrm{COPD}^{4,5}$.

Chronic inflammation in COPD can persist even after smoking cessation ${ }^{6}$. The long-term effects of CS include airway structural damage that leads to secondary bacterial infection in the airways ${ }^{6}$. This bacterial colonization persistently provides antigens that induce airway 
inflammation. For example, lipopolysaccharide (LPS), which is produced in response to infection, activates airway epithelial cells to release $\mathrm{IL}-8^{7}$ and the resultant neutrophilic inflammation contributes to the development of COPD ${ }^{8}$.

In addition to chronic inflammation, oxidative stress caused by reactive oxygen species (ROS) contributes to the pathogenesis of COPD. The most well-known source of ROS in the pathogenesis of COPD is CS, which causes tissue destruction and DNA damage ${ }^{9}$. Moreover, CS reinforces oxidative stress by triggering the generation of additional ROS, further augmenting inflammation ${ }^{2,10}$. In addition to the crosstalk that occurs between the inflammatory processes and oxidative stress pathways, inflammation and protease enzymatic activities are also likely to be mutually reinforcing. On the one hand, inflammatory cells, especially neutrophils, secrete various proteases, including neutrophil elastase (NE), proteinase 3, myeloperoxidase, and cathepsin. On the other, NE increases the recruitment of neutrophils in response to the presence of chemotactic fragments that are produced by the cleavage of proteins in the extracellular matrix and from the destruction of lung tissue ${ }^{11,12}$. Moreover, we have previously reported that crosstalk occurs between the catalytic processes of NE and those leading to IL-8 production after exposure to $\mathrm{CS}^{13,14}$. Therefore, inflammation, oxidative stress, and protease-antiprotease imbalance constantly interact with each other to drive disease progression.

In contrast to CS, chronic inflammation caused by bacterial infection and its interaction with proteolytic activity remain unclear. In fact, IL-8, neutrophils, and NE activity are further increased in the bronchoalveolar lavage fluid of COPD patients with persistent airway bacterial infections ${ }^{8}$. However, the crosstalk between proteases and inflammation induced by bacterial infection has not been elucidated. Therefore, in this study, the aim was to evaluate the effect of NE on LPS-induced inflammatory responses and to determine the molecular mechanism in bronchial epithelial cells.

\section{Materials and methods}

\section{Study subjects and animals}

For animal experiments, female 6-week-old C57BL/6 wild-type (WT) mice were purchased from KOATECH (Pyeongtaek, South Korea). All animal experiments were approved by the Institutional Animal Care and Use Committee (No. 19-0220-S1A1[1]) of Seoul National University Hospital, Seoul, South Korea.

Normal human bronchial epithelial cells (HBECs) (BEAS-2B cells) were maintained in defined keratinocyte serum-free medium (Gibco by Life Technologies, Grand Island, NY, USA) at $37^{\circ} \mathrm{C}$ and $5 \% \mathrm{CO}_{2}$. Primary HBECs were provided by participants who underwent bronchoscopies for diagnostic or therapeutic purposes.
These cells were obtained from the lobar and subsegmental bronchial lumens by bronchial brushing. All patients consented to providing their epithelial cells, and the Institutional Review Board of Seoul National University Hospital approved the study protocol (IRB No. H-1602-108-742).

\section{Reagents}

Human sputum NE was acquired from Elastin Product Company (Owensville, MO, USA). The NE was dissolved in a solution containing 50\% $0.02 \mathrm{M} \mathrm{NaOAc}(\mathrm{pH}$ 5) and 50\% glycerol. LPS, phenylmethylsulfonyl fluoride (PMSF), and rosiglitazone were obtained from Sigma-Aldrich (St. Louis, MO, USA). The selective elastase inhibitor Elaspol was purchased from Ono Pharmaceutical Co., Ltd. (Osaka, Japan). The anti-IkB $\alpha$ antibody was obtained from Cell Signaling Technology (Danvers, MA, USA). Anti-peroxisome proliferator-activated receptor gamma (PPAR $\gamma$ ), anti-TLR4, anti-p65, anti-NE, and anti-glyceraldehyde 3-phosphate dehydrogenase (GAPDH) antibodies were all purchased from Santa Cruz Biotechnology (Santa Cruz, CA, USA).

\section{Protein extraction and western blot analysis}

Total cellular proteins were extracted using $1 \times$ cell lysis buffer (Cell Signaling Technology). Membrane proteins were isolated using a membrane protein extraction kit (Thermo Fisher Scientific, Waltham, MA, USA). Protein concentrations were determined using the Bradford protein assay according to the manufacturer's instructions (Bio-Rad, Hercules, CA, USA). Proteins were separated by 4-12\% sodium dodecyl sulfate-polyacrylamide gel electrophoresis (SDS-PAGE) and then transferred to nitrocellulose membranes. The membranes were blocked with $5 \%$ skim milk blocking buffer for $1 \mathrm{~h}$, followed by overnight incubation at $4{ }^{\circ} \mathrm{C}$ with primary antibodies in blocking buffer. The membranes were washed in washing buffer three times, followed by incubation with secondary antibodies for $1 \mathrm{~h}$. After successive washes, the membranes were developed using a SuperSignal West Pico Chemiluminescent kit (Thermo Fisher Scientific).

\section{Flow cytometry}

Cells were incubated with anti-TLR4 phycoerythrin (PE) or anti-immunoglobulin (Ig) G PE in $200 \mu \mathrm{L}$ of incubation buffer for $45 \mathrm{~min}$. Unreacted PE-TLR4 antibodies were removed. Cell-associated PE-conjugated antibodies were analyzed by flow cytometry using a FACSCalibur or a FACSCanto flow cytometer (BD Biosciences, San Jose, CA, USA).

\section{Quantification of IL-8 secretion}

The levels of IL-8 in cell culture supernatants were measured using a commercially available human IL-8/ CXCL8 DuoSet enzyme-linked immunosorbent assay 
(ELISA) kit (R\&D System, Minneapolis, MN, USA) according to the manufacturer's instructions.

\section{Transfection of plasmid vectors}

Transfections of the control vector and the pAdTrackCMV-PPARy expression vector were performed using a Neon Transfection System (Thermo Fisher Scientific) according to the manufacturer's instructions.

\section{Real-time quantitative polymerase chain reaction (RT-qPCR)}

Total RNA was isolated using an RNeasy kit (Qiagen, Hilden, Germany). Total RNA $(1 \mu \mathrm{g})$ was reversetranscribed to cDNA using a reverse transcription system (Promega, Madison, WI, USA). Power SYBR Green PCR Master Mix (Applied Biosystems, Foster City, CA, USA) was used for amplification. The primers used in the study were as follows: IL-8 (fwd: 5'-GCA GCT CTG TGT GAA GGT GC-3', rev: 5'-TCT GCA CCC AGT TTT CCT TG-3'); PPAR $\gamma$ (fwd: $5^{\prime}$-TGT GGG GAT AAA GCA TCA GGC-3', rev: 5'-CCG GCA GTT AAG ATC ACA CCT AT-3'); TLR4 (fwd: 5'-CTG CAA TGG ATC AAA GGA CCA-3', rev: 5'TTA TCT GAA GGT GTT GCA CAT TCC-3'); and GAPDH (fwd: 5'-GAA GGT GAA GGT CGG AGT C-3', rev: 5'-GAA GAT GGT GAT GGG ATT TC-3').

\section{Immunofluorescent labeling}

Cells were grown in $35 \mathrm{~mm}$ dishes, fixed in methanol for $10 \mathrm{~min}$ and washed three times. The cells were then incubated with the anti-p65 antibody diluted 1:100 in 3\% bovine serum albumin (BSA) for $24 \mathrm{~h}$. The cells were subsequently incubated with an Alexa Fluor 555 antibody (Thermo Fisher Scientific) diluted 1:100 in 3\% BSA for $30 \mathrm{~min}$. After successive washes, the cells were analyzed using EVOS digital color fluorescence microscopy (Thermo Fisher Scientific).

\section{PPARY fragmentation and quantification}

Cell lysates were incubated with either the vehicle control (50\% glycerol, $50 \% 0.02 \mathrm{M} \mathrm{NaOAc}, \mathrm{pH}$ ) ) or the $\mathrm{NE}$ at $37^{\circ} \mathrm{C}$ for $30 \mathrm{~min}$. The reaction was terminated by adding an excess of western blot sample buffer. The samples were subjected to western blot analysis to quantify the concentrations of PPAR $\gamma$ and NE.

\section{Intratracheal administration of elastase}

C57BL/6 WT mice were anesthetized, and saline or elastase was administered intratracheally $(0.5 \mathrm{U}$ in $100 \mu \mathrm{L}$ of saline). The mice were sacrificed 1 day after elastase administration, and the lungs were isolated for immunohistochemical labeling.

\section{Immunohistochemistry}

Lung tissues were fixed, embedded, sectioned at a thickness of $4 \mu \mathrm{m}$, and placed on slides for labeling using the Discovery XT automated immunohistochemistry staining system (Ventana Medical Systems, Inc., Tucson, AZ, USA). Tissue sections were deparaffinized and rehydrated. For antigen retrieval, a Cell Conditioning 1 (Ventana Medical Systems) standard ( $\mathrm{pH} 8.4$ buffer containing tris/borate/EDTA) was used. The sections were incubated with the anti-PPAR $\gamma$ antibody at $37^{\circ} \mathrm{C}$ for $32 \mathrm{~min}$, washed, and incubated with a secondary antibody for $20 \mathrm{~min}$. After successive washes, the slides were incubated with 3,3-diaminobenzidine $\mathrm{H}_{2} \mathrm{O}_{2}$ substrate at $37^{\circ} \mathrm{C}$ for $8 \mathrm{~min}$, followed by counterstaining with hematoxylin and a bluing reagent. Stained cells were observed under a microscope (EVOS XL Core Cell Imaging System, Thermo Fisher Scientific).

\section{Statistical analysis}

The data were analyzed with a two-tailed unpaired $t$-test or Mann-Whitney $U$ test to assess significant differences between groups. All statistical analyses were performed using GraphPad Prism software (San Diego, CA, USA). A $P$-value $<0.05$ was considered to be statistically significant.

\section{Results}

NE synergistically augments LPS-induced IL-8 production in lung epithelial cells

The effect of NE on LPS-induced IL-8 production was assessed. As expected, stimulation with LPS $(1 \mu \mathrm{g} / \mathrm{mL})$ increased the number of IL- 8 mRNA transcripts and protein concentrations in BEAS-2B cells and primary HBECs (Fig. 1). While treatment with NE alone did not affect the level of IL-8, pretreatment with NE for $4 \mathrm{~h}$ augmented the LPS-induced production of IL-8 in both BEAS-2B cells and primary HBECs (Fig. 1).

\section{Neither the TLR4 nor the IKB/NF-KB pathway is involved in NE-mediated augmentation of LPS-induced IL-8 production}

Since it is well known that LPS first binds to TLR4, which then activates the I $\mathrm{kB} / \mathrm{NF}-\mathrm{kB}$ pathway to ultimately result in IL-8 production ${ }^{15}$, whether the TLR4 or IкB/NF$\kappa B$ pathway was involved in mediating the NE-mediated synergistic increase in LPS-induced IL-8 production was tested. TLR4 expression or its affinity for LPS should be increased if TLR4 is involved in mediating the synergistic increase in LPS-induced IL-8 production. However, while NE treatment increased the mRNA levels of TLR4 in BEAS-2B cells and HBECs (Supplementary Fig. 1a, b), it decreased the protein concentration of TLR4 in both the total cellular extracts and membrane fractions (Supplementary Fig. 1c-f). LPS stimulation led to ІкB $\alpha$ degradation and the subsequent nuclear translocation of $\mathrm{p} 65$ (an NF- $\kappa B$ subunit); this outcome was not affected by NE treatment (Fig. 2a, b). ІкB $\alpha$ phosphorylation was also not 

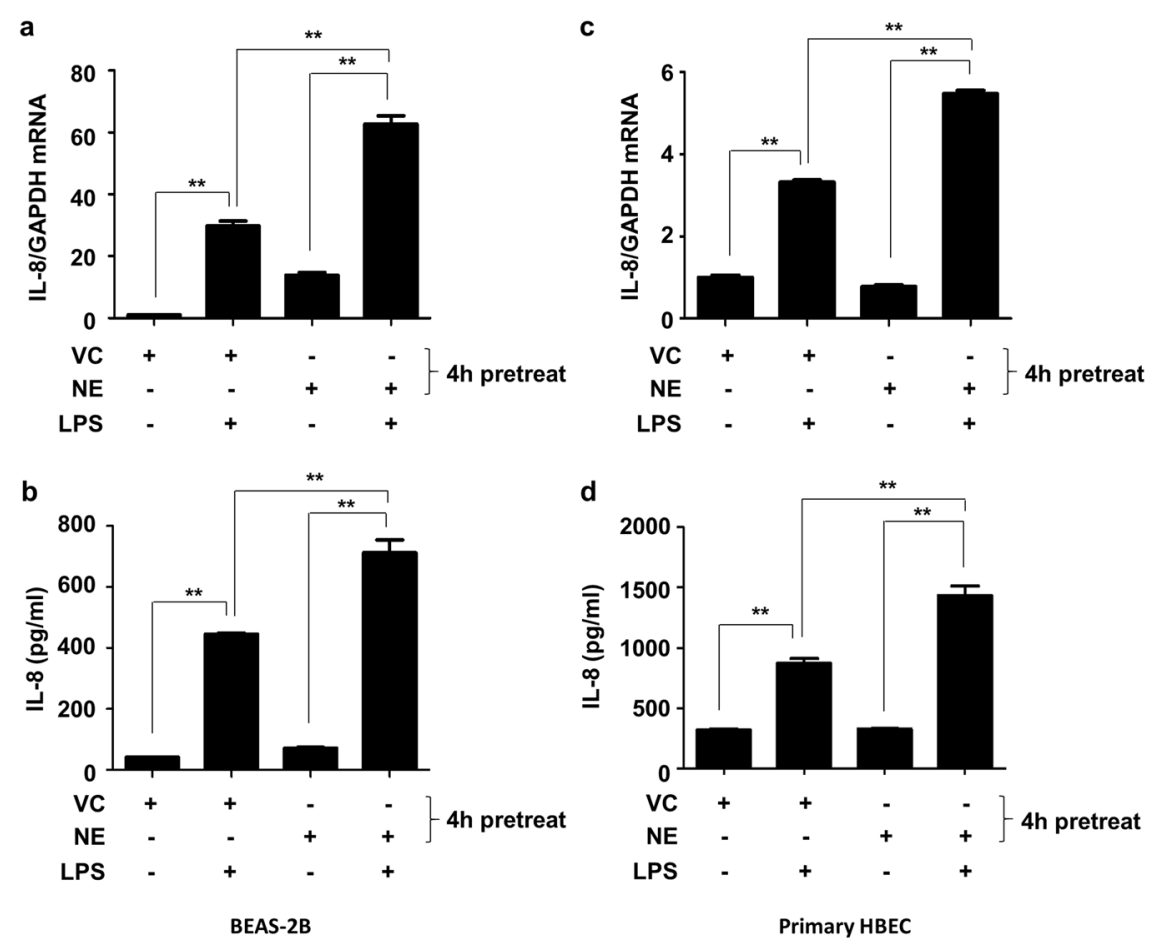

Fig. 1 NE synergistically augments the LPS-induced production of IL-8 in lung epithelial cells. BEAS-2B cells (a, b) and primary HBECS (c, d) were pretreated with VC or NE $(1 \mathrm{U} / \mathrm{mL})$ for $4 \mathrm{~h}$ and then stimulated with LPS $(1 \mu \mathrm{g} / \mathrm{mL})$ for $16 \mathrm{~h}$ in the presence or absence of VC or NE. Total RNA was isolated from BEAS-2B cells (a) and HBECs (c), and quantitative real-time PCR analysis of IL-8 and GAPDH was performed. The levels of IL-8 in the supernatants of BEAS-2B cells (b) and HBECs (d) were measured by ELISA. The data represent mean \pm SD; ${ }^{* *} P<0.05$. Abbreviations: NE, neutrophil elastase; LPS, lipopolysaccharide; IL-8, interleukin 8; HBECs, human bronchial epithelial cells; VC, vehicle control; RNA, ribonucleic acid; PCR, polymerase chain reaction; GAPDH, glyceraldehyde 3-phosphate dehydrogenase; ELISA, enzyme-linked immunosorbent assays; SD, standard deviation.

affected by NE treatment (data not shown). Thus, the TLR4 and IKB/NF-kB pathways are unlikely to be involved in mediating the NE-mediated synergistic increase in LPSinduced IL-8 production.

\section{NE causes the degradation of PPARY}

PPAR $\gamma$, a ligand-activated transcription factor in the nucleus, has been reported to suppress the transcriptional activity of NF- $\mathrm{kB}$, leading to a decrease in IL-8 production ${ }^{16}$. While the level of PPARY mRNA was not affected by NE treatment in BEAS-2B cells (Fig. 3a), the concentration of PPAR $\gamma$ protein decreased (Fig. 3b, c). Coincubation with either a serine protease inhibitor (PMSF) or an elastase inhibitor (Elaspol) blocked the NE-induced decrease in PPARY concentration (Fig. 3d). Thus, NE downregulates PPAR $\gamma$ through proteolytic degradation.

PPARy downregulation is responsible for the NE-mediated synergistic increase in LPS-induced IL-8 production

Next, the role of NE-mediated PPARy downregulation in mediating the synergistic increase in LPS-induced IL-8 production was assessed. Stimulation of PPARy by rosiglitazone suppressed the NE-mediated synergistic increase in
LPS-induced IL-8 production (Fig. 4a). Moreover, the overexpression of PPAR $\gamma$, which is not degraded by NE, also suppressed the NE-mediated synergistic increase in LPS-induced IL-8 production (Fig. 4b, c). These data indicate that PPARy downregulation by NE is responsible for the synergistic increase in LPS-induced IL-8 production.

\section{PPARy expression levels are decreased in airway epithelial} cells in both elastase-treated mice and in patients with COPD

Finally, we evaluated whether these in vitro findings could also be observed in vivo. PPARy expression levels were lower in lung homogenates from elastase-treated mice than in those from saline-treated mice (Fig. 5a, b). Immunohistochemical staining showed that the decrease in PPARy expression was prominent in lung epithelial cells (Fig. 5c). In accordance with the findings in mice, the expression of PPARy in primary HBECs isolated from the large and small airways of COPD patients was significantly lower than that in cells from healthy neversmokers and healthy smokers (Fig. 6). The detailed characteristics of these patients are shown in Supplementary Table 1. 


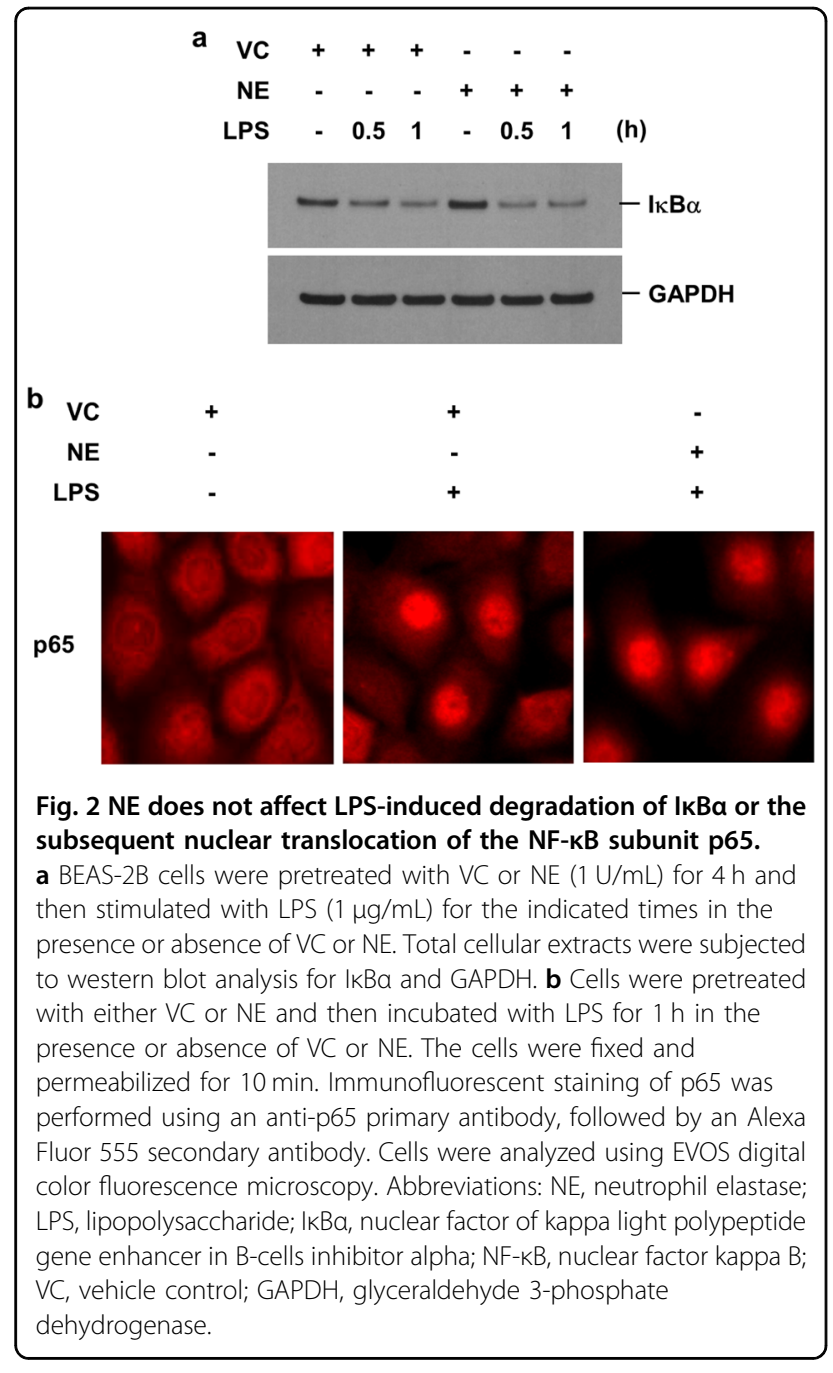

\section{Discussion}

The mechanisms of the crosstalk between protease activity and inflammatory processes in HBECs have not been clearly elucidated. This study showed that NE synergistically increases LPS-induced inflammation through PPAR $\gamma$ degradation in HBECs. In accordance with previous studies ${ }^{17-19}$, our study showed that LPS upregulated IL-8 mRNA and protein expression in BEAS$2 B$ cells and primary HBECs; interestingly, our findings also revealed that this upregulated expression was augmented by NE, indicating increased de novo IL-8 protein synthesis. IL-8 is a well-known chemotactic factor that leads to the recruitment of neutrophils, which release NE; this release causes structural damage through extracellular matrix degradation in $\mathrm{COPD}^{20}$. The augmentation of LPS-induced IL-8 production by NE suggests that $\mathrm{NE}$ potentiates inflammation in addition to directly damaging the tissue. This synergistic interaction could explain the more prominent and perpetual inflammation that occurs when COPD is exacerbated ${ }^{21}$. Although these

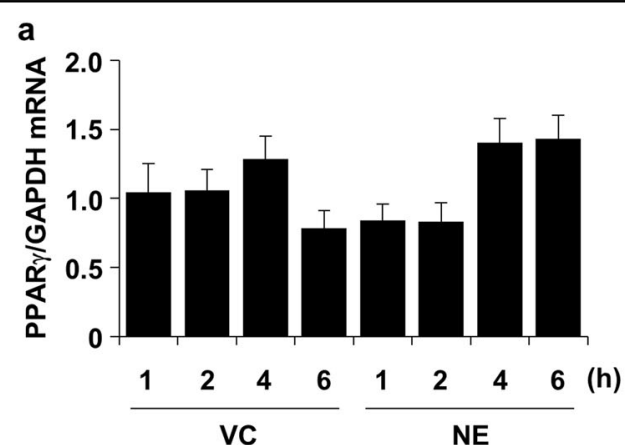

b
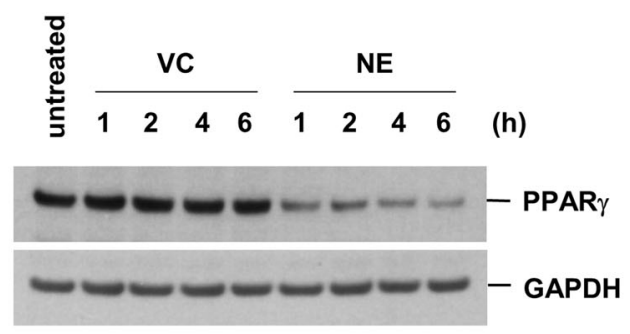

C

in vitro fragmentation assay $\left(37^{\circ} \mathrm{C}, 30 \mathrm{~min}\right)$

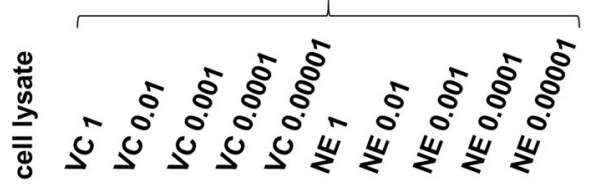

d

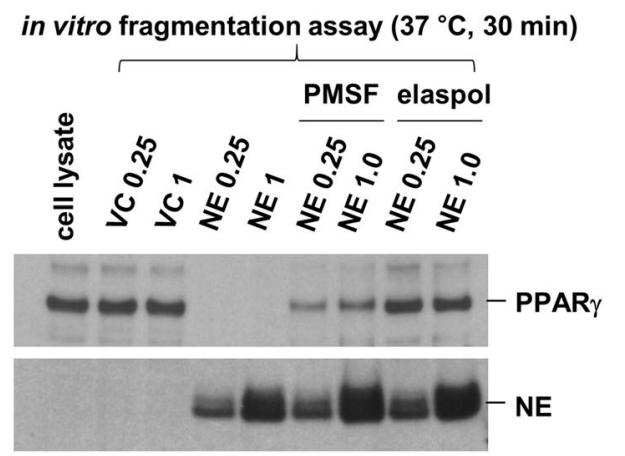

Fig. 3 NE downregulates PPARy through proteolytic degradation. BEAS-2B cells were treated with $\mathrm{VC}$ or $\mathrm{NE}(1 \mathrm{U} / \mathrm{mL})$ for the indicated times. a Total RNA was isolated, and quantitative real-time PCR analysis of PPARY and GAPDH was performed. The data represent mean \pm SD. $\mathbf{b}$ Total cellular extracts were subjected to western blot analysis for PPARy and GAPDH protein quantification. $\mathbf{c}, \mathbf{d}$ Cell lysates were incubated with VC or $\mathrm{NE}(0.00001,0.0001,0.001,0.01,0.25$, and $1 \mathrm{U} / \mathrm{mL})$ in the presence or absence of a serine protease inhibitor (PMSF, $1 \mathrm{mM}$ ) or an elastase inhibitor (Elaspol, $100 \mu \mathrm{g} / \mathrm{mL}$ ) at $37^{\circ} \mathrm{C}$ for $30 \mathrm{~min}$. The reaction was terminated by adding an excess of sample buffer; subsequently, the samples were subjected to western blot analysis for PPARy quantification. Abbreviations: $\mathrm{NE}$, neutrophil elastase; PPARY, peroxisome proliferator-activated receptor gamma; VC, vehicle control; RNA, ribonucleic acid; PCR, polymerase chain reaction; GAPDH, glyceraldehyde 3-phosphate dehydrogenase; SD, standard deviation; PMSF, phenylmethylsulfonyl fluoride. 


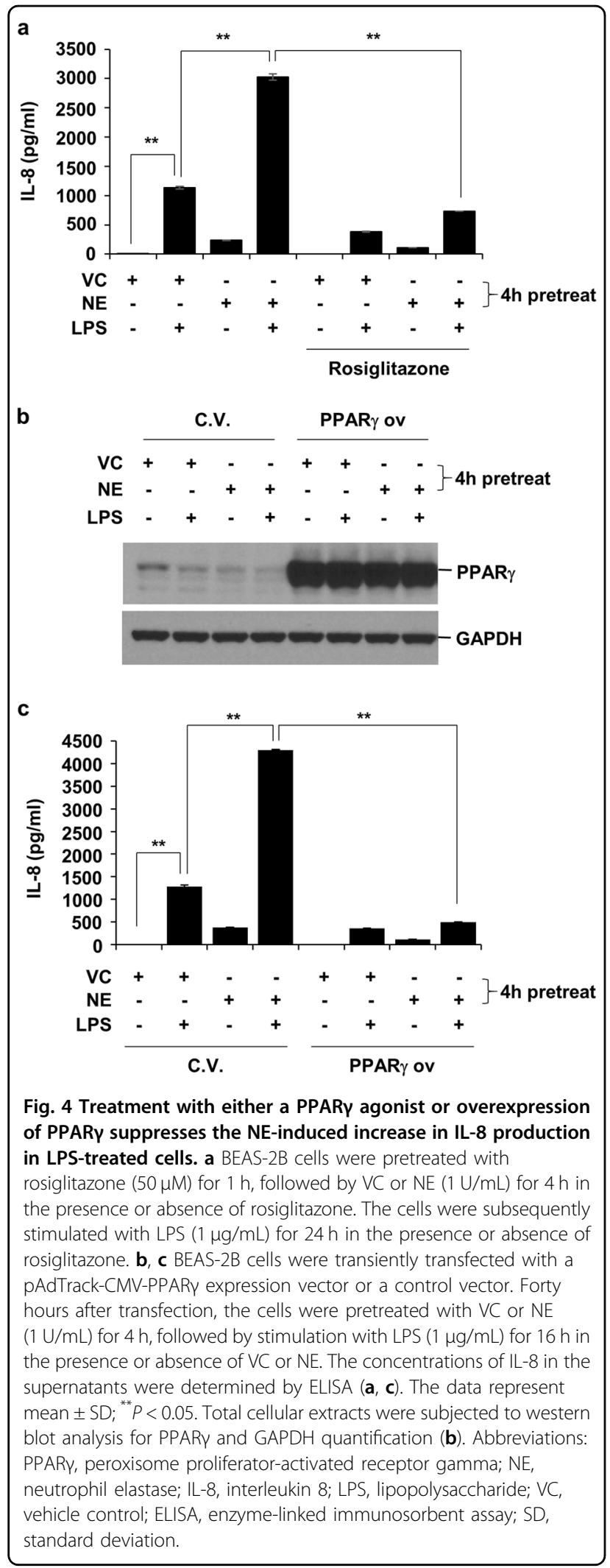

a

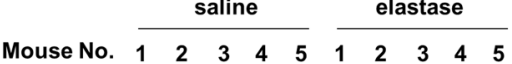

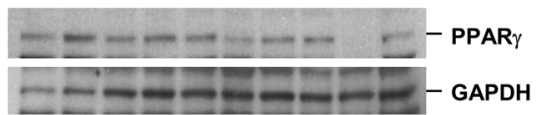

b

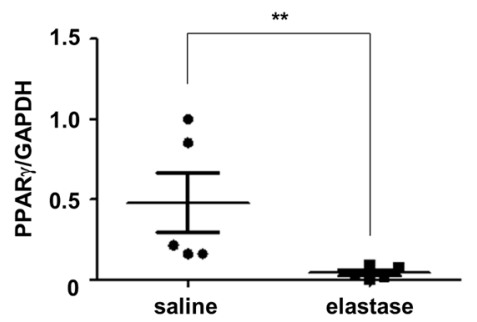

C

IHC : PPAR $\gamma$

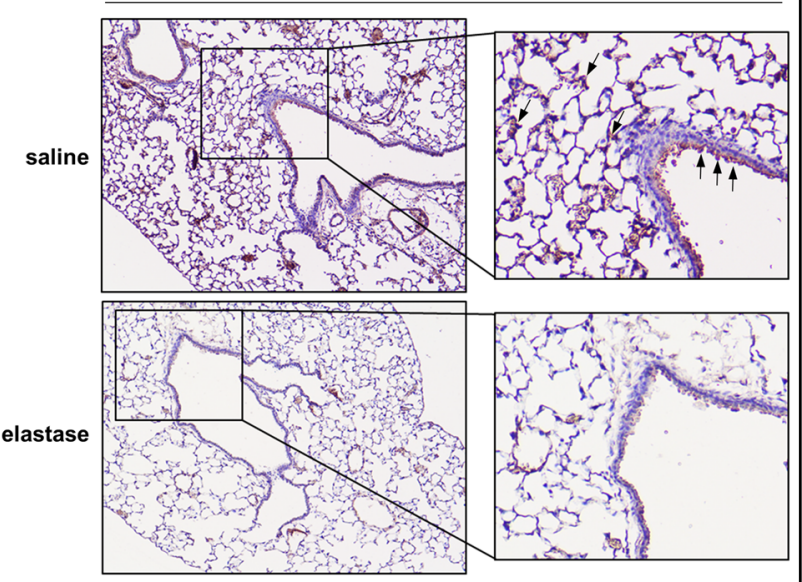

Fig. 5 The expression levels of PPARY are decreased in the lung epithelial cells of elastase-treated mice. a-c C57BL/6 mice were intratracheally administered either saline or elastase $(0.5 \mathrm{U}$ in $100 \mu \mathrm{L}$ saline). The mice ( $n=5$ per group) were sacrificed 1 day after elastase treatment, and the lungs were isolated. Total lung protein lysates from the mice were subjected to western blot analysis for PPARy and GAPDH quantification (a). The gel data were quantified using Scion image densitometry (b). The data represent mean $\pm \mathrm{SE} ;{ }^{* *} P<0.05$. c PPARy immunohistochemical analysis in lung tissues collected from mice. Arrows indicate epithelial cells stained with PPARY.

Abbreviations: PPARy, peroxisome proliferator-activated receptor gamma; GAPDH, glyceraldehyde 3-phosphate dehydrogenase; SE, standard error.

findings concerning the crosstalk between inflammation and protease activity may not be generalizable, it seems likely that the augmentation is mutual; indeed, the mutual augmentation of IL-8 has also been reported between NE and CS extract (CSE) $)^{13,14}$.

Although NE augmented LPS-induced inflammation, the precise mechanism has yet to be clarified. When LPS 

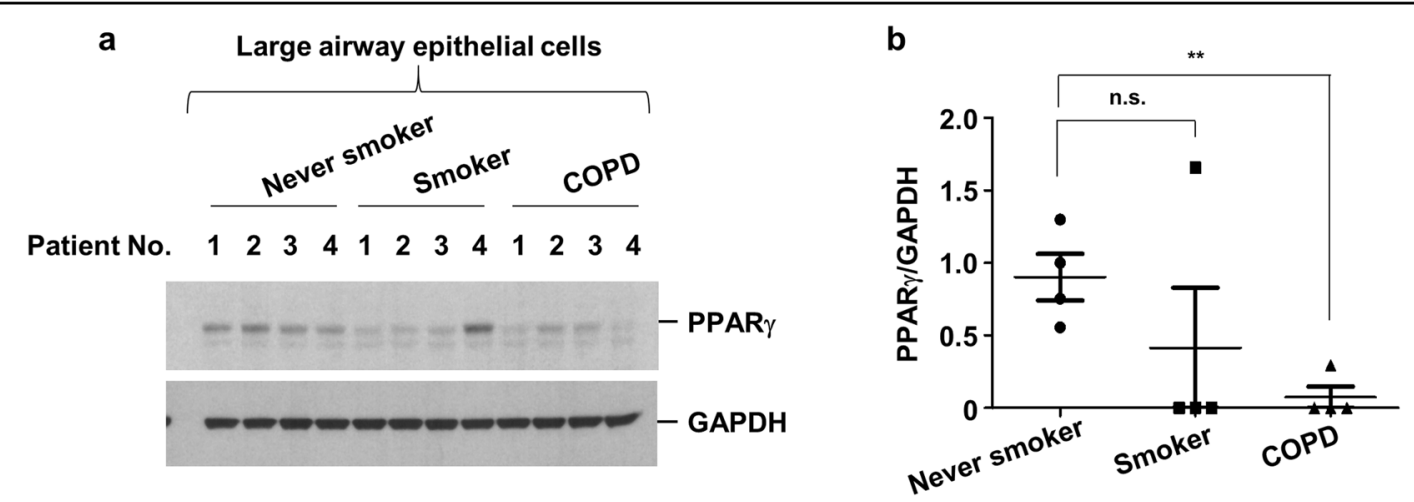

C
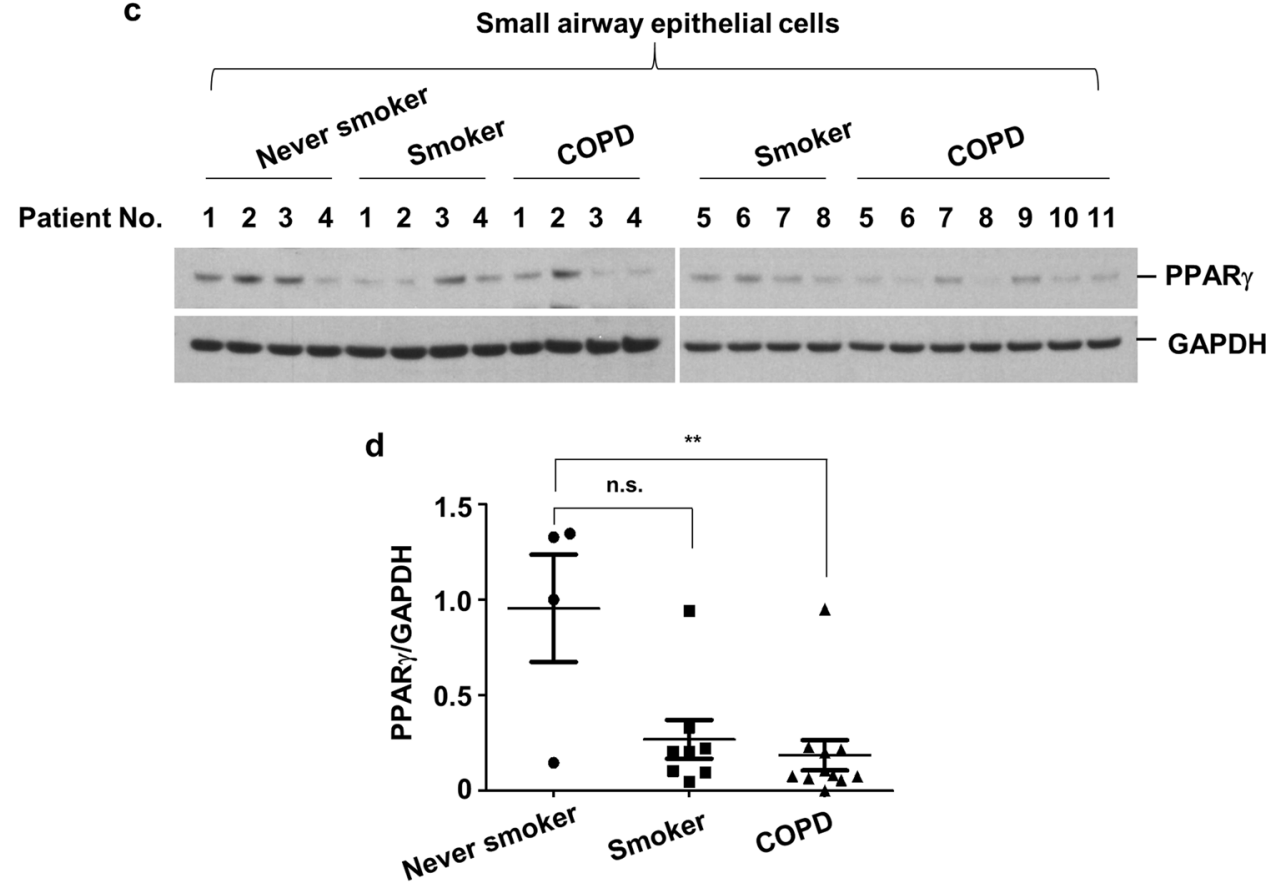

Fig. 6 PPARy levels are decreased in the airway epithelial cells of COPD patients. $\mathbf{a}$, $\mathbf{b}$ Large airway epithelial cells were obtained from neversmokers $(n=4)$, smokers $(n=4)$ and COPD patients $(n=4)$. $\mathbf{c} \mathbf{d}$ Small airway epithelial cells were acquired from never-smokers $(n=4)$, smokers $(n=$ 8) and COPD patients $(n=11)$. Total cell lysates were subjected to western blot analysis for PPARY and GAPDH quantification (a, $\mathbf{c})$. The gel data were quantified using Scion image densitometry $\left(\mathbf{b}\right.$, d). The data represent mean $\pm \mathrm{SE}_{;}{ }^{* *} P<0.05$. Abbreviations: PPARY, peroxisome proliferator-activated receptor gamma; COPD, chronic obstructive pulmonary disease; GAPDH; glyceraldehyde 3-phosphate dehydrogenase; SE, standard error.

binds to TLR4, signal transduction is initiated to synthesize IL-8 via the IkB/NF- $\mathrm{KB}$ pathway ${ }^{22}$; therefore, the first focus was to assess changes in TLR-4 expression. In contrast to the increased TLR4 expression induced by LPS $^{23}$, TLR4 expression decreased following NE administration in total cellular extracts and membrane fractions, which is consistent with a previous report ${ }^{24}$. Next, whether the $\mathrm{I} \kappa \mathrm{B} / \mathrm{NF}-\mathrm{kB}$ pathway was involved in the NEmediated augmentation of LPS-induced IL-8 production was evaluated. In this study, LPS stimulation led to ІкB $\alpha$ degradation and the subsequent nuclear translocation of p65 (an NF-kB subunit), as described in a previous report $^{22}$; this effect was not altered by NE treatment.
Thus, it seems likely that neither the TLR4 pathway nor the $\mathrm{I} \kappa \mathrm{B} / \mathrm{NF}-\kappa \mathrm{B}$ pathway is involved in the NE-mediated augmentation of LPS-induced inflammation. Unlike a previous study, which reported that the one mediated augmentation of CSE-induced IL-8 production was mediated through the extracellular signal-regulated kinase (ERK) pathway ${ }^{13}$, in this study, ERK activation was not involved in the augmenting effect of NE (data not shown), suggesting that the molecular mechanisms might be stimulus-specific.

As neither the TLR4 pathway nor the IкB/NF- $\mathrm{B}$ pathway was involved in the NE-mediated augmentation of LPSinduced IL- 8 production, as well as the fact that the 
increased IL-8 production was due to de novo protein synthesis, we next hypothesized that NE might affect the transcription of IL- 8 within the nucleus. Because transcription of IL- 8 in the nucleus could be modulated by PPAR $\gamma$ in human lung epithelial cells ${ }^{16}$, the effects of PPAR $\gamma$ were examined. PPAR $\gamma$ is a ligand-activated transcription factor that belongs to the nuclear hormone receptor family. Following translocation into the nucleus, PPAR $\gamma$ interferes with NF- $\mathrm{KB}$ and activator protein 1 (AP-1), inhibiting the transcription of proinflammatory cytokines, including IL-8 $8^{25}$. NE induced the proteolytic degradation of PPAR $\gamma$. Moreover, the NE-mediated augmentation of LPS-induced IL-8 production was reversed by treatment with either the PPARY agonist rosiglitazone or overexpression of PPARy. Taken together, these findings indicate that PPAR $\gamma$ downregulation is responsible for the NE-mediated augmentation of LPSinduced IL-8 production. In accordance with these findings, PPAR $\gamma$ agonists have been shown to attenuate proinflammatory gene expression in the lungs of mice ${ }^{26}$. In addition, PPAR $\gamma$ agonists also ameliorate elastase-induced emphysema in mice ${ }^{27}$. Based on these findings, the PPAR $\gamma$ agonist has been recently spotlighted as a new candidate for COPD treatment ${ }^{28}$.

This study demonstrated that the downregulation of PPAR $\gamma$ resulted in NE-mediated augmentation of LPSinduced IL-8 production in vitro; to extend these findings, the study aimed to determine if these effects translated to in vivo experiments in animals and in human tissues. In accordance with the in vitro findings, the expression of PPAR $\gamma$ was decreased in the lung homogenates of elastasetreated mice. This decrease was most pronounced within bronchial epithelial cells, based on immunohistochemical labeling experiments. Furthermore, it was also found that the PPARy levels in HBECs obtained from patients with COPD were lower than those in cells from healthy neversmokers or healthy smokers, while current smoking status itself did not affect PPAR $\gamma$ levels (data not shown). Thus, the in vitro and in vivo results were consistent.

Beyond the pathogenesis of stable COPD, the role of PPARY agonists in COPD exacerbation, which is most frequently caused by respiratory infections, has been suggested. Large population studies from the United States and Taiwan have reported that the use of PPARY agonists reduced the risk of COPD exacerbation ${ }^{29,30}$; likewise, PPAR $\gamma$ agonists also decreased the risk of exacerbation in patients with asthma ${ }^{31}$. CS-induced neutrophilia followed by nontypeable Haemophilus influenza infection was attenuated by rosiglitazone in mice ${ }^{32}$. Although these studies suggest the beneficial effects of PPAR $\gamma$ agonists on COPD exacerbation, the exact mechanisms are still unknown. A future study will focus on investigating the role of PPAR $\gamma$ agonists in a model of COPD exacerbation.
To the best of our knowledge, this is the first study to demonstrate the mechanism by which NE augments LPSinduced IL-8 production in bronchial epithelial cells and to elucidate its role in the pathogenesis of COPD. In conclusion, a vicious cycle of mutual augmentation occurs between protease activity and inflammatory processes through PPARY degradation; these interactions play a role in the pathogenesis of COPD. These results suggest that the restoration of PPARY homeostasis could be a new therapeutic strategy in the treatment of COPD.

\section{Acknowledgements}

This work was supported by the Seoul National University Hospital Research Fund (Grant number: 04-2018-0680). Seoul National University Hospital Research Fund (Grant number: 04-2018-0680).

Conflict of interest

The authors declare no competing interests.

\section{Publisher's note}

Springer Nature remains neutral with regard to jurisdictional claims in published maps and institutional affiliations.

Supplementary information The online version contains supplementary material available at https://doi.org/10.1038/s12276-021-00626-7.

Received: 2 February 2021 Revised: 15 March 2021 Accepted: 16 March 2021.

Published online: 21 May 2021

\section{References}

1. Bateman, E. D., Reddel, H. K., van Zyl-Smit, R. N. \& Agusti, A. The asthma-COPD overlap syndrome: towards a revised taxonomy of chronic airways diseases? Lancet Respir. Med. 3, 719-728 (2015).

2. Fischer, B. M., Pavlisko, E. \& Voynow, J. A. Pathogenic triad in COPD: oxidative stress, protease-antiprotease imbalance, and inflammation. Int. J. Chron. Obstruct. Pulmon. Dis. 6, 413 (2011).

3. Barnes, P. J. et al. Chronic obstructive pulmonary disease. Nat. Rev. Dis. Prim. 1, 15076 (2015).

4. Brusselle, G. G., Joos, G. F. \& Bracke, K. R. New insights into the immunology of chronic obstructive pulmonary disease. Lancet 378, 1015-1026 (2011).

5. Boots, A. W. et al. Neutrophils augment LPS-mediated pro-inflammatory signaling in human lung epithelial cells. Biochim. Biophys. Acta 1823, 1151-1162 (2012).

6. King, P. T., MacDonald, M. \& Bardin, P. G. Bacteria in COPD; their potential role and treatment. Transl. Respir. Med. 1, 1-9 (2013).

7. Comer, D. M., Kidney, J. C., Ennis, M. \& Elborn, J. S. Airway epithelial cell apoptosis and inflammation in COPD, smokers and nonsmokers. Eur. Respir. J. 41, 1058-1067 (2013).

8. Sethi, S., Maloney, J., Grove, L., Wrona, C. \& Berenson, C. S. Airway inflammation and bronchial bacterial colonization in chronic obstructive pulmonary disease. Am. J. Respir. Crit. Care Med. 173, 991-998 (2006).

9. Deslee, G. et al. Cigarette smoke induces nucleic-acid oxidation in lung fibroblasts. Am. J. Respir. Cell. Mol. Biol. 43, 576-584 (2010).

10. Rahman, I. \& Adcock, I. Oxidative stress and redox regulation of lung inflammation in COPD. Eur. Respir. J. 28, 219-242 (2006).

11. Hunninghake, G. et al. Elastin fragments attract macrophage precursors to diseased sites in pulmonary emphysema. Science 212, 925-927 (1981).

12. Weathington, N. M. et al. A novel peptide CXCR ligand derived from extracellular matrix degradation during airway inflammation. Nat. Med. 12, 317-323 (2006).

13. Lee, K. H., Lee, C. H., Jeong, J., Jang, A. H. \& Yoo, C. G. Neutrophil elastase differentially regulates interleukin 8 (IL-8) and vascular endothelial growth 
factor (VEGF) production by cigarette smoke extract. J. Biol. Chem. 290, 28438-28445 (2015)

14. Lee, K. H. et al. Cigarette smoke extract enhances neutrophil elastase-induced IL-8 production via proteinase-activated receptor-2 upregulation in human bronchial epithelial cells. Exp. Mol. Med. 50, 1-9 (2018).

15. Hoffmann, E., Dittrich-Breiholz, O., Holtmann, H. \& Kracht, M. Multiple control of interleukin-8 gene expression. J. Leukoc. Biol. 72, 847-855 (2002).

16. Neri, T. et al. Role of NF-KB and PPAR-y in lung inflammation induced by monocyte-derived microparticles. Eur. Respir. J. 37, 1494-1502 (2011).

17. Mayer, A. K. et al. Differential recognition of TLR-dependent microbial ligands in human bronchial epithelial cells. J. Immunol. 178, 3134-3142 (2007).

18. Khair, O. et al. Effect of Haemophilus influenzae endotoxin on the synthesis of IL-6, IL-8, TNF-alpha and expression of ICAM-1 in cultured human bronchial epithelial cells. Eur. Respir. J. 7, 2109-2116 (1994).

19. Monick, M. M. et al. Respiratory syncytial virus up-regulates TLR4 and sensitizes airway epithelial cells to endotoxin. J. Biol. Chem. 278, 53035-53044 (2003).

20. Stockley, R. A. Neutrophils and the pathogenesis of COPD. Chest 121, 151S-155S (2002).

21. Hoenderdos, K. \& Condliffe, A. The neutrophil in chronic obstructive pulmonary disease. Too little, too late or too much, too soon? Am. J. Respir. Cell. Mol. Biol. 48, 531-539 (2013).

22. Lu, Y. C., Yeh, W. C. \& Ohashi, P. S. LPS/TLR4 signal transduction pathway. Cytokine 42, 145-151 (2008).

23. Jiang, Q. et al. Protective effects of polydatin on lipopolysaccharide-induced acute lung injury through TLR4-MyD88-NF-KB pathway. Int. Immunopharmacol. 29, 370-376 (2015).
24. Devaney, J. M. et al. Neutrophil elastase up-regulates interleukin-8 via toll-like receptor 4. FEBS Lett. 544, 129-132 (2003).

25. Becker, J., Delayre-Orthez, C., Frossard, N. \& Pons, F. Regulation of inflammation by PPARs: a future approach to treat lung inflammatory diseases? Fundam. Clin. Pharmacol. 20, 429-447 (2006).

26. Shan, M. et al. Agonistic induction of PPARy reverses cigarette smoke-induced emphysema. J. Clin. Invest. 124, 1371-1381 (2014).

27. Boo, H. J. et al. $\sqcup-529$, a partial peroxisome proliferator-activated receptor gamma (PPARY) agonist and adenosine A3 receptor agonist, ameliorates elastase-induced pulmonary emphysema in mice. Arch. Pharm. Res. 43 540-552 (2020).

28. Lakshmi, S. P., Reddy, A. T. \& Reddy, R. C. Emerging pharmaceutical therapies for COPD. Int. J. Chron. Obstruct. Pulmon. Dis. 12, 2141 (2017)

29. Rinne, S. T. et al. Thiazolidinediones are associated with a reduced risk of COPD exacerbations. Int. J. Chron. Obstruct. Pulmon. Dis. 10, 1591 (2015).

30. Wang, M. T. et al. Use of antidiabetic medications and risk of chronic obstructive pulmonary disease exacerbation requiring hospitalization: a disease risk score-matched nested case-control study. Respir. Res. 21, 1-18 (2020).

31. Rinne, S. T. et al. Thiazolidinediones and the risk of asthma exacerbation among patients with diabetes: a cohort study. Allergy Asthma Clin. Immunol. 10, 1-6 (2014).

32. Morissette, M. C., Shen, P., Thayaparan, D. \& Stämpfli, M. R. Impacts of peroxisome proliferator-activated receptor- $\gamma$ activation on cigarette smoke-induced exacerbated response to bacteria. Eur. Respir. J. 45, 191-200 (2015) 